\title{
Correction to: Emotional Intelligence as an Ability: Theory, Challenges, and New Directions
}

\author{
Marina Fiori and Ashley K. Vesely-Maillefer
}

\section{Correction to:}

Chapter 2 in: K. V. Keefer et al. (eds.), Emotional Intelligence in Education, The Springer Series on Human Exceptionality, https://doi.org/10.1007/978-3-319-90633-1_2

Chapter 2 of this book has been converted to open access and the copyright holder has been changed to 'The Author(s)'.

The book has also been updated with these changes.

The updated online version of this chapter can be found at https://doi.org/10.1007/978-3-319-90633-1_2 